\title{
Suppression of GABAergic transmission in the spinal dorsal horn induces pain-related behaviour in a chicken model of spina bifida
}

\author{
Md. Sakirul Islam Khan ${ }^{1}$, Hiroaki Nabeka ${ }^{1}$, Farzana Islam ${ }^{1}$, Tetsuya Shimokawa ${ }^{1}$, Shouichiro Saito ${ }^{2}$, \\ Tetsuya Tachibana ${ }^{3}$, Seiji Matsuda ${ }^{1}$ \\ ${ }^{1}$ Department of Anatomy and Embryology, Graduate School of Medicine, Ehime University, Toon, Ehime, Japan, ${ }^{2}$ Laboratory of Veterinary \\ Anatomy, Faculty of Applied Biological Sciences, Gifu University, Yanagido, Gifu, Japan, ${ }^{3}$ Department of Agrobiological Science, Faculty \\ of Agriculture, Ehime University, Matsuyama, Japan
}

\begin{abstract}
Spina bifida aperta (SBA), one of the most common congenital malformations, causes various neurological disorders. Pain is a common complaint of patients with SBA. However, little is known about the neuropathology of SBA-related pain. Because loss of $\gamma$-aminobutyric acid GABAergic neurons in the spinal cord dorsal horn is associated with pain, we hypothesised the existence of crosstalk between SBA-related pain and alterations in GABAergic transmission in the spinal cord. Therefore, we investigated the kinetics of GABAergic transmission in the spinal cord dorsal horn in a chicken model of SBA. Neonatal chicks with SBA exhibited various pain-like behaviours, such as an increased number of vocalisations with elevated intensity (loudness) and frequency (pitch), reduced mobility, difficulty with locomotion, and escape reactions. Furthermore, the chicks with SBA did not respond to standard toe-pinching, indicating disruption of the spinal cord sensorimotor networks. These behavioural observations were concomitant with loss of GABAergic transmission in the spinal cord dorsal horn. We also found apoptosis of GABAergic neurons in the superficial dorsal horn in the early neonatal period, although cellular abnormalisation and propagation of neurodegenerative signals were evident at middle to advanced gestational stages. In conclusion, ablation of GABAergic neurons induced alterations in spinal cord neuronal networks, providing novel insights into the pathophysiology of SBA-related pain-like complications.
\end{abstract}

Key words: spina bifida, chicken model, pain-related behaviour, GABAergic transmission, apoptosis.

\section{Introduction}

Spina bifida aperta (SBA), a neural-tube defect (NTD) that causes lifelong neurological complications, develops in approximately 1 per 1000 neonates worldwide [8]. SBA is primarily characterised by defective fusion of the neural tube, which causes in utero deformities in the exposed spinal $[9,12,28,32]$. Such spinal cord deformities lead to varying degrees of motor and/or sensory deficits, resulting in neurological disorders such as spinal ataxia, paralysis of the legs, problems with bowel and bladder control, and pain complications $[26,28,35,41]$. NTD-induced

\section{Communicating author}

Md. Sakirul Islam Khan, MS, PhD, Department of Anatomy and Embryology, Ehime University Graduate School of Medicine,

Toon 791-0295, Ehime, Japan, phone: 81-89-960-5231, fax: 81-89-960-5233, e-mail: sakirul@m.ehime-u.ac.jp, sakirul.khan@gmail.com 
pain is a common complaint of SBA patients of any age $[7,40,41]$. Individuals with SBA frequently have various risk factors for pain, such as musculoskeletal deformities, clogged/infected shunts, urinary tract infections, bowel problems, and suboptimal positioning $[2,36]$. In addition to physiological pain, patients with SBA often undergo surgeries, which can cause pathological pain. The literature on pain in SBA is largely inconclusive in terms of prevalence, aetiologies, and prevention or treatment strategies $[2,15,36]$. The gross pathological lesion suggested that SBA-induced pain may be related to incomplete development of the neural arch, and nature fills the defect with dense fibrous tissue [15]. However, little is known about the pathophysiology of spinal cord and pain in SBA. Thus, research in animal models of SBA is needed to better understand the underlying cellular and molecular mechanisms and to develop novel therapeutic interventions.

The pain experienced by people with NTD may be nociceptive or neuropathic or a combination thereof [41]. A loss of inhibitory transmission in the spinal cord dorsal horn is important in the development of chronic pain. Spinal cord injury-induced loss of $\gamma$-aminobutyric acid GABAergic neurons - the principle inhibitory interneurons - in the superficial dorsal horn causes persistent pain [13,14,23,42]. Calciumbinding proteins, particularly those in GABAergic subpopulations, have received much attention due to their robust, developmentally regulated, and cell type-specific expression in the nervous system, and they have emerged as effective markers to identify neurons in pain networks [34]. These facts led us to postulate the existence of crosstalk between SBArelated pain complications and alteration in GABAergic transmission in the spinal cord dorsal horn. Accordingly, we explored the kinetics of GABAergic transmission in the spinal cord dorsal horn from gestation to posthatching in a chicken model of SBA to obtain pathophysiological data on the sequence of events associated with SBA-like neurological disorders $[17,29,38]$.

\section{Material and methods}

\section{Animals}

The fertilised eggs of chickens (Gallus gallus; Mori Hatchery, Kagawa, Japan) were incubated in a commercial incubator (Showa Furanki, Saitama, Japan) at 37.8 $\pm 0.2^{\circ} \mathrm{C}$ with $60 \%$ relative humidity to obtain embryos at developmental stages 17-21 inclusive on gestational days 2.5-3.5. The developmental stage of each embryo was determined using the developmental table of Hamburger and Hamilton [11]. The embryos were divided into two groups: the SBA group, in which the roof plate of the neural tube was incised; and the normal control group, in which the neural tube was left intact. The hatched chicks were raised in a room maintained at $30^{\circ} \mathrm{C}$ with continuous illumination and were fed a commercial diet (crude protein, 24\%; metabolisable energy, 3050 kcal/kg; Toyohashi Feed Mills Co. Ltd., Toyohashi, Japan) with water available ad libitum. Because neonatal SBA chicks have difficulty consuming food, they were gavaged a feed slurry (tube feeding) at a mass of $4.0 \%$ of their body weight into the crop four times daily. The feed slurry was made by mixing $40 \%$ powdered diet with $60 \%$ distilled water on a weight basis. The animal experimental protocols were approved by the Committee on the Ethics of Animal Experiments of the Ehime University Graduate School of Medicine, Toon, Japan (No. 05A27-10).

\section{Surgical manipulation to generate spina bifida aperta chicks}

To produce SBA chicks, surgical manipulation of the neural plate was carried out as described previously $[17,29]$. Briefly, the eggshell and amnion were opened and placed under a stereomicroscope to determine the developmental stage of the embryo. Next, the roof plate of the neural tube was incised longitudinally, starting at the level of the cranial margin of the $26^{\text {th }}$ somite, which forms the sixth and seventh thoracic segments, using a custom-made microknife $[18,19]$. The incision extended caudally for a distance equivalent to the length of seven somites and was made by inserting the microknife into the neural tube to approximately half the depth of the tube. The roof plate was incised, and care was taken not to damage other parts of the neural tube. After the incision was made and the surgical manipulation was complete, the shell window was closed using transparent adhesive tape, and the eggs were re-incubated at 37.8 $\pm 0.2^{\circ} \mathrm{C}$ with $60 \%$ relative humidity.

\section{Behavioural assessments}

For behavioural assessments, the SBA and control chicks used at postnatal day (PD)-0 were also used at PD-2, PD-4, and PD-10. To characterise pain-related behavioural changes, the spontaneous activities of the normal and SBA chicks were video- 
taped at 8:00 and 20:00 for 30 min at PD-0, PD-2, PD-4, and PD-10 and scored offline. The intensity and number of vocalisations - the primary indicators of pain in birds $[25,33]$ - were characterised by two independent observers. The intensity of vocalisation (Frequency Analyzer 2.0; free software) and number of vocalisations per $10 \mathrm{~min}$ were assessed in the SBA and normal chicks.

To elicit a sensorimotor reaction, forceps were used to conduct a standard pinch test of the toes on both legs of the normal and SBA chicks; the tests were repeated three times per chick. The results were considered conclusive and a normal reaction was recorded only if an identical response was obtained on at least three toes in each leg in three consecutive tests. The reactions of the chicks were videotaped and the leg withdrawal reflex, jumping, and vocalisation behaviours were scored offline by two independent observers. Behaviour was analysed in six chicks per group at each age.

\section{Sample collection and tissue preparation}

Spinal cord sections from the open defect area (lumbosacral region) from SBA chicks, and from a similar location in the normal chicks, were collected on embryonic days (ED)-14, ED-18, PD-2, PD-4, and PD-10. The incisions of the roof plates of the neural tubes of SBA embryos were confirmed by observing open defects on the backs of the chicks, and the induction of SBA in the chicks post-hatching was confirmed by observing open defects on the backs of the chicks with leg dysfunction ([17]; Fig. 1A; Suppl. Video 1). Six chicks from each group at each age were transcardially perfused with a fixative solution containing 4\% paraformaldehyde with $0.5 \%$ glutaraldehyde in $0.1 \mathrm{M}$ phosphate-buffered saline (PBS). Next, the spinal cord was removed from the location of the open defect (exposed area, lesion location). Spinal tissue samples from the normal control chicks were collected from locations similar to the tissue collection locations of SBA chicks. The collected tissues were immersed in $4 \%$ paraformaldehyde overnight at $4^{\circ} \mathrm{C}$, dehydrated, and embedded in paraffin.

\section{Immunohistochemical staining}

The avidin-biotin complex method was used to analyse GABAergic immunoreactivities. Briefly, spinal cord sections $(7 \mu \mathrm{m})$ from the location of the open defect (exposed area) of the lumbosacral cord in the SBA chicks, and from a similar location in the normal chicks, were deparaffinised, rehydrated, and treated with PBS containing 10\% methanol and 3\% hydrogen peroxide $\left(\mathrm{H}_{2} \mathrm{O}_{2}\right)$ for 10 min. After rinsing with PBS, the spinal cord sections were treated with $5 \%$ bovine serum albumin, $1 \%$ normal goat serum, $0.1 \%$ fish gelatine, and $0.1 \% \mathrm{NaN}_{3}$ in PBS for $1 \mathrm{~h}$ followed by incubation with the primary antibodies, rabbit polyclonal anti-GABA (1 : 1500; Sigma, St. Louis, MO) overnight at $4^{\circ} \mathrm{C}$, or mouse monoclonal anti-parvalbumin (PV) (1 : 500; Sigma), mouse monoclonal anti-calbindin-D-28K (CB), or mouse monoclonal anti-calretinin (CR) (1 : 500; SWANT, Bellinzona, Switzerland) for $60 \mathrm{~h}$ at $4^{\circ} \mathrm{C}$. Next, the sections were washed three times for 10 min each in PBS and reacted with the secondary antibodies for $2 \mathrm{~h}$ at room temperature. After rinsing three times for $10 \mathrm{~min}$ each in PBS, the avidin-biotin-peroxidase complex (1 : 300; Dako, Glostrup, Denmark) was applied for $1 \mathrm{~h}$ at room temperature. The sections were immersed in 3,3-diaminobenzidine (DAB) (Sigma) containing $0.0033 \% \mathrm{H}_{2} \mathrm{O}_{2}$ for approximately $10 \mathrm{~min}$. After rinsing with distilled water, the sections were dehydrated, mounted, and visualised under a light microscope (Nikon, Tokyo, Japan).

Antibody specificity was tested using a negative staining procedure with normal rabbit or mouse IgG (1 : 250; Dako, Glostrup, Denmark) instead of the primary antibodies; the samples were processed as described above. No specific signals were found using normal rabbit or mouse IgG (data not shown).

\section{Quantitative analysis of GABAergic neurons}

For quantitative analysis of GABA-expressing neurons, serial coronal sections $(7 \mu \mathrm{m})$ from the location of the open defect (exposed area) of the lumbar cord in the SBA chicks, and from a similar location in the normal chicks, were stained with a rabbit polyclonal anti-GABA antibody (1 : 1500; Sigma). The tissues were observed under a Nikon Eclipse E800 light microscope, and images were acquired using a charge-coupled device (CCD) camera attached to the microscope (Nikon Digital Sight DS-L2). For analysis of GABAexpressing neurons, all GABA-immunopositive cells with a rounded profile in superficial dorsal horn laminae I-III were considered and counted. The total num- 
A

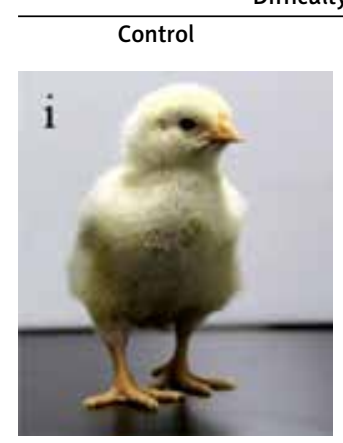

B

$\frac{\text { Number of vocalisations }}{\square \text { Control } \quad \text { SBA }}$

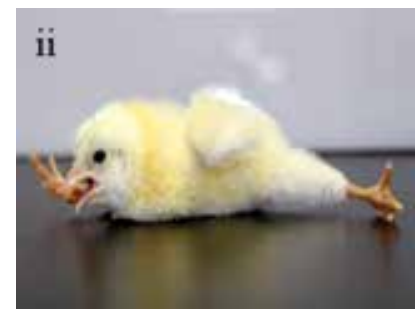

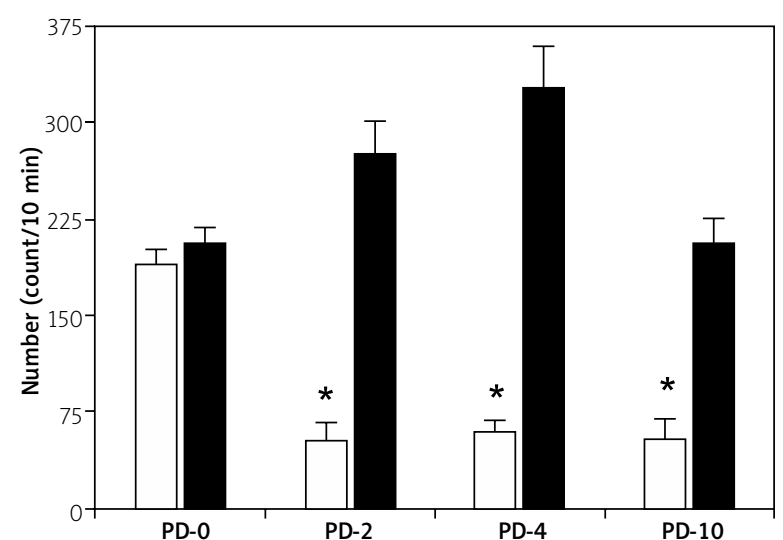

C

PD-0
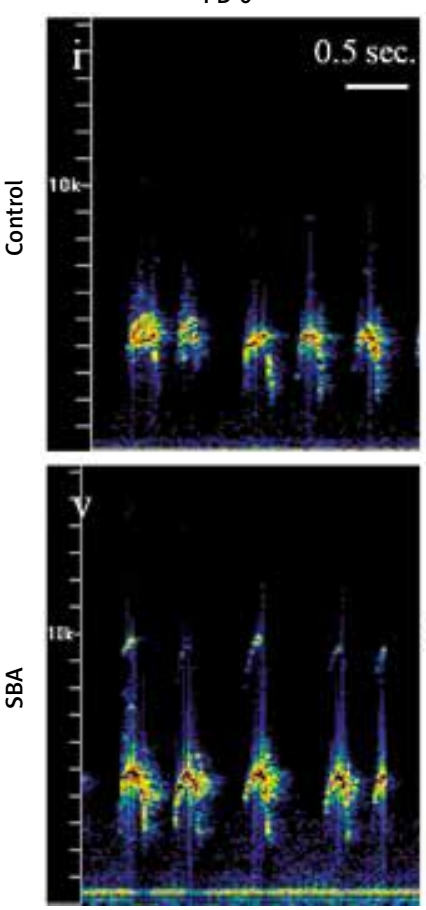

PD-2
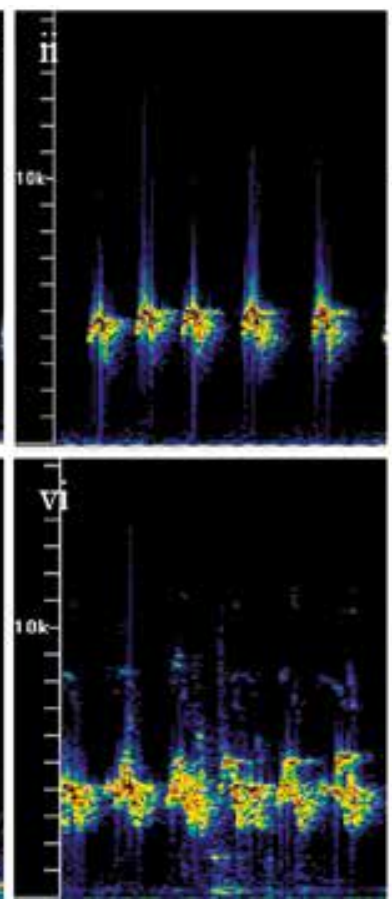

PD-4
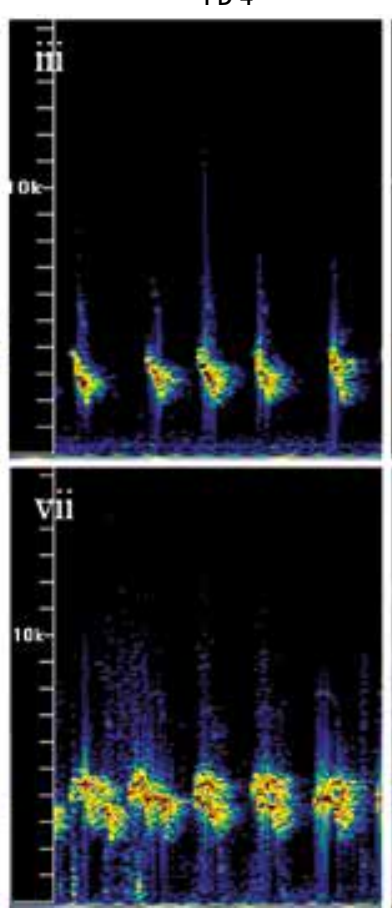

PD-10

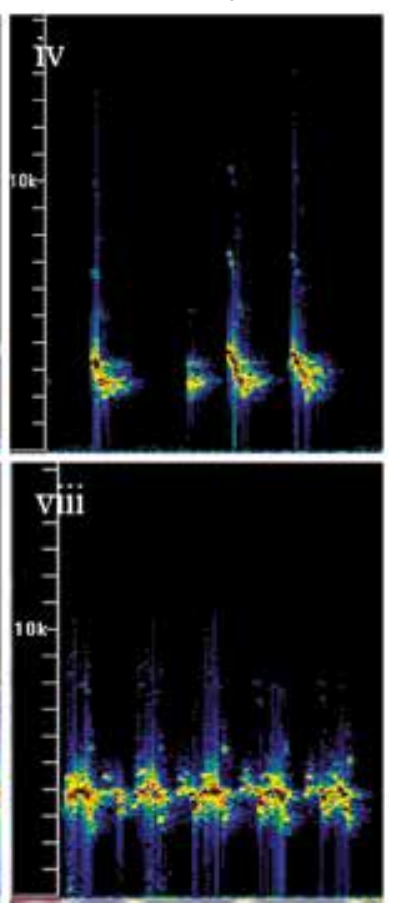

Fig. 1. Pain-like behavioural changes in spina bifida aperta (SBA) chicks. A) Representative photographs of the phenotypes of normal and SBA chicks. The control chicks were able to move freely (i) whereas SBA chicks moved with severe difficulty (ii). B) Numbers of vocalisations in normal and SBA neonatal chicks. C) The intensity (loudness) and frequency (pitch) of vocalisations in normal and SBA neonatal chicks. The data in Figure $1 \mathrm{~B}$ are means $\pm \mathrm{SEM} ; n=6$ per group at each age. ${ }^{*}$ Significantly different from the SBA group $(p<0.001)$, two-way ANOVA and post hoc Tukey's test.

ber of neurons in the superficial dorsal horn on each side of the spinal cord was calculated and averaged across six cross sections per chick at each age; each group comprised six chicks of each age.
For quantitative analysis of PV-, CB-, and CR-expressing neurons, serial coronal sections were stained with mouse monoclonal anti-PV (1 : 500; Sigma), mouse monoclonal anti-CB, or mouse monoclonal 
anti-CR (1 : 500; SWANT) antibody, and PV-, CB-, and CR-immunopositive cells were enumerated as described above.

\section{Measurement of staining intensity}

The GABAergic immunoreactivities in the dorsal horn lamina I-III of the open defect in the spinal cord of SBA chicks, and at a similar location in normal chicks, were measured using ImageJ software (National Institutes of Health, Bethesda, MD, USA). To correct for background, three background intensity readings were taken per image. These readings were averaged and subtracted from the signal intensity to yield the protein staining intensity. The intensity data are presented as the mean \pm SEM. Six random sections per chick at each age in each group were analysed for quantification; each group comprised six chicks of each age.

\section{Immunofluorescence staining}

Immunofluorescence staining was performed as described previously [17]. For double immunofluorescence staining, the sections were incubated for $60 \mathrm{~h}$ at $4^{\circ} \mathrm{C}$ in a solution containing rabbit polyclonal anti-GABA (1 : 3000; Sigma) plus mouse monoclonal anti-CB or mouse monoclonal anti-CR (1 : 1000; SWANT) antibodies; and rabbit polyclonal anti-caspase 3 (1 : 1000; Bioss) plus mouse monoclonal anti-CB (1 : 1000; SWANT) antibodies. After washing in PBS, the sections were treated for $2 \mathrm{~h}$ at room temperature with an Alexa Fluor 546-conjugated goat anti-rabbit IgG $(\mathrm{H}+\mathrm{L})$ (1 : 1000; Invitrogen) or an Alexa Fluor 488-conjugated goat anti-mouse IgG $(\mathrm{H}+\mathrm{L})$ (1 : 1000; Invitrogen) and 4',6-diamidino-2-phenylindole (DAPI), washed with PBS, mounted with Vectashield (Vector Laboratories), and visualised using a Nikon A1 confocal microscope equipped with a 100× objective lens (Nikon).

\section{Statistical analysis}

Statistical analysis was performed on the mean values, and data are reported as the mean \pm SEM. The data were subjected to two-way analysis of variance (ANOVA) with the Tukey-Kramer post hoc test, and $p$-values $<0.05$ were considered to indicate statistical significance.

\section{Results \\ Assessment of behavioural disorders}

Various neurological complications were evident in neonatal chicks with SBA (Suppl. Video 1). Difficulty during locomotion (Fig. 1A) and increased numbers of vocalisations (Fig. 1B), with greater intensity (loudness) and frequency (pitch; Fig. 1C), were observed in SBA chicks compared with control chicks during the early neonatal period. Chicks with SBA also showed reduced confidence in mobility, escape reactions, anxiety, fear or restlessness, and inappetence. These behavioural changes are considered primary indicators of pain in birds [25,33], indicating that the chicks with SBA experienced pain.

The sensorimotor reactions of the chicks are summarised in Table I. In normal chicks, toe-pinching resulted in withdrawal of the limb, jumping, and vocalisation (Suppl. Video 2). The responses were consistent in control chicks at PD-0, PD-2, PD-4, and PD-10; therefore, the pattern was considered a normal sensorimotor reaction. In the first few hours after hatching (PD-0), toe-pinching elicited relatively normal reactions in SBA chicks compared to age-matched control chicks. However, commencing on PD-2, the sensorimotor reactions gradually decreased; there was almost no response at PD-10 (Table I; Suppl. Video 1), indicating disruption of the sensorimotor networks in the chicks with SBA.

Table I. Assessment of sensorimotor reactions in spina bifida aperta (SBA) chicks

\begin{tabular}{|c|c|c|c|c|c|c|}
\hline \multirow[t]{3}{*}{ Age } & \multicolumn{6}{|c|}{ Sensorimotor reactions by toe-pinching (total score: 5 per parameter) } \\
\hline & \multicolumn{3}{|c|}{ Control } & \multicolumn{3}{|c|}{ SBA } \\
\hline & Leg withdrawal & Vocalisation & Jump & Leg withdrawal & Vocalisation & Jump \\
\hline PD-0 & 4 & 4 & 5 & 5 & 4 & 5 \\
\hline PD-2 & 5 & 5 & 5 & 3 & 5 & 2 \\
\hline PD-4 & 5 & 5 & 5 & 2 & $5^{*}$ & 1 \\
\hline PD-10 & 4 & 3 & 4 & 1 & $5^{\star}$ & 0 \\
\hline
\end{tabular}

A 5-point scoring system was used to grade sensorimotor reactions (leg withdrawal reflex, jumping, and vocalisations) after toe-pinching in normal control and SBA chicks at the early neonatal stage. Sensorimotor reactions were graded as no reaction (0 points), minimal reaction (1-2 points), reduced reaction (3-4 points), and normal reaction (5 points). *Difficult to distinguish toe-pinching-induced vocalisation reaction, as the SBA chicks were experienced persistent vocalisation. 

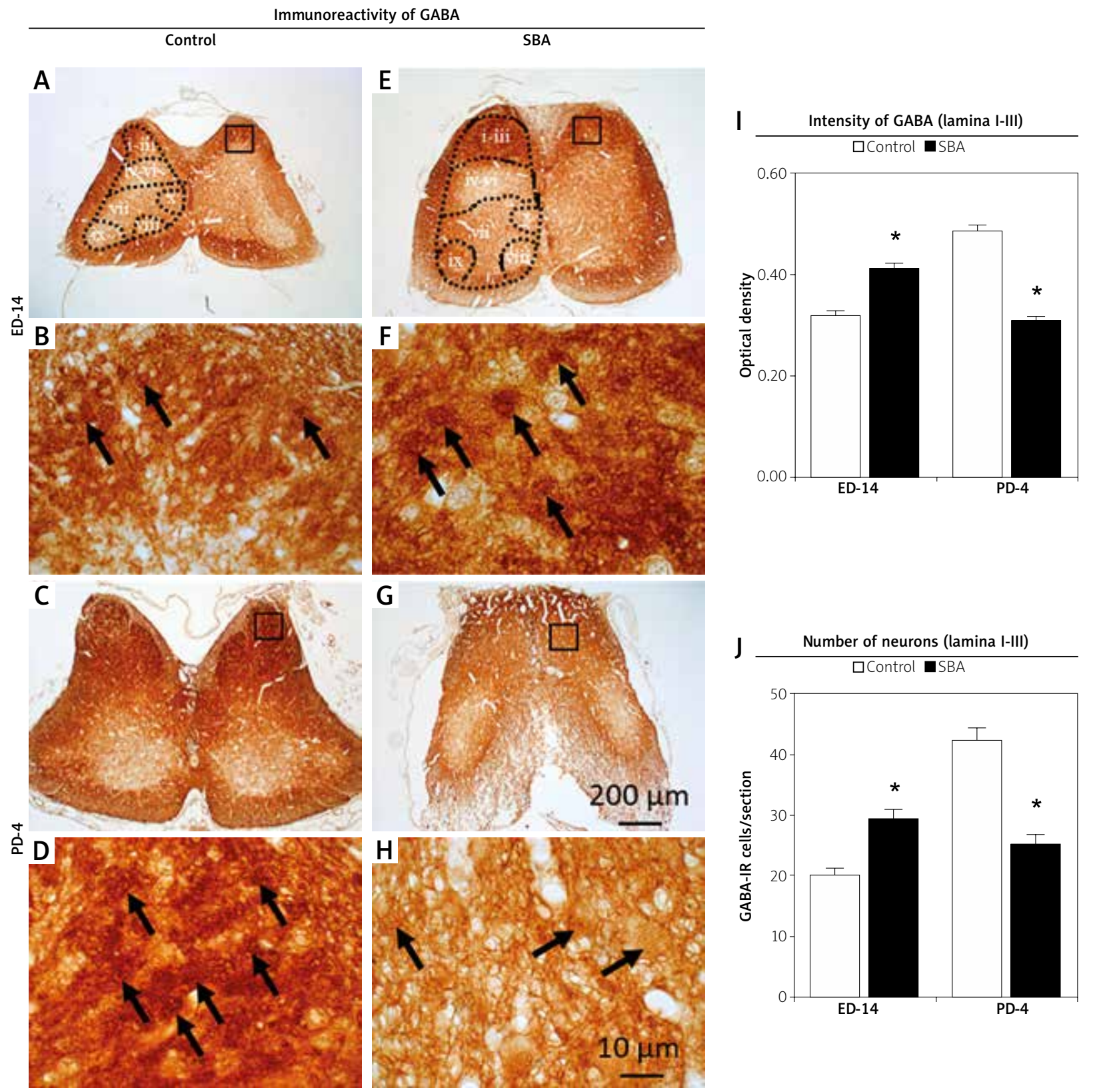

Fig. 2. Postnatal loss of $\gamma$-aminobutyric acid (GABA) immunoreactivity in the spinal cord dorsal horns of spina bifida aperta (SBA) chicks. A-H) Immunohistochemical analysis of the expression and localisation of GABA in the spinal cord open defect area in SBA chicks, and at a similar location in normal chicks, at ED-14 and PD-4. Representative images of the paraffin-embedded spinal cords of control (A-D) and SBA (E-H) chicks. I) GABA immunoreactivity in the dorsal horn laminae I-III of control and SBA chicks measured using ImageJ software. The optical density (OD) was calculated using the following formula: $\mathrm{OD}=\left(\log _{10}\right.$ [incident light/transmitted light]). Six random sections per chick at each age and for each group were analysed. J) Number of GABA-immunopositive cells in the spinal cord dorsal horn laminae I-III of control and SBA chicks. The data in Figure $2 \mathrm{I}$ and $\mathrm{J}$ are means $\pm \mathrm{SEM} ; n=6$ in each group at each age. ${ }^{*}$ Significantly different from the SBA group at each age $(p<0.01)$, two-way ANOVA and post hoc Tukey's test. Arrows indicate GABA-immunopositive cells. 


\section{Expression of GABA in the dorsal horn of the spinal cord}

The GABA immunoreactivity in exposed spinal cord in embryonic and neonatal chicks is shown in Figure 2. GABA immunoreactivity was detected in the spinal cord, predominantly in dorsal horn laminae I-III. Such GABA immunoreactivity in avian species is in line with mammalian studies of GABA distribution in the spinal cord [24]. GABA immunoreactivity was higher in SBA chicks than in normal chicks at the embryonic stage (ED-14). In contrast, GABA immunoreactivity was lower in SBA chicks at the neonatal stage (PD-4) compared to not only age-matched normal chicks but also SBA chicks at the embryonic stage. In fact, at ED-14, GABA expression was significantly higher in SBA chicks than in age-matched control chicks, whereas the opposite pattern was evident at PD-4 (Fig. 2I). At ED-14, the number of GABA-immunopositive cells was significantly higher in dorsal horn laminae I-III of SBA chicks than in the control chicks, but the number decreased at PD-4 (Fig. 2J).

\section{Expression of calcium-binding GABAergic subpopulations in the spinal cord dorsal horn}

Figures 3, 4, and 5 show the immunoreactivities of the PV, $C R$, and $C B$ calcium-binding GABAergic subpopulations, respectively. PV-positive neurons were predominantly detected in the ventral horn area, but also in the dorsal horn (Fig. 3). The overall density of PV-immunopositive fibres in the spinal cord increased with increasing developmental stage in the normal chicks (Fig. 3A-D) but not in the SBA chicks (Fig. 3E-H). Similarly, the number of PV-positive neurons in dorsal horn laminae I-III at ED-14 was greater in SBA chicks than in normal chicks; however, after hatching (at P4), the opposite pattern was evident (Fig. 3J). PV immunoreactivity at ED-14 was significantly greater in the SBA chicks than in age-matched control chicks; the opposite pattern was evident at the neonatal stage (Fig. 3l).

$C R$ immunoreactivity in the spinal cord of the SBA and normal chicks is shown in Figure 4. A few intensely labelled CR-immunopositive cells were observed in both the normal and SBA chicks at the embryonic stage, and this was relatively prominent in dorsal horn laminae I-III of the SBA chicks. Although the CR immunoreactivity of cells and fibres increased in the spinal cords of neonatal normal chicks (Fig. 4A-D), CR immunoreactivity was lower in SBA chicks at PD-4 (Fig. 4E-H). Additionally, the number and staining intensity (Fig. $4 \mathrm{l}$, J) of CRexpressing neurons in dorsal horn laminae I-III at PD-4 were significantly reduced in SBA chicks compared to the control chicks.

CB immunoreactivity in the spinal cords of SBA and normal chicks is shown in Figure 5. CB-immunopositive cells were detected in the dorsal and ventral horns of the spinal cord. In the ventral horn, strongly CB-immunoreactive small neurons were distributed in lamina VII of both the normal and SBA chicks; in mammals, these are reportedly Renshaw cells $[3,4]$. The number and staining intensity of CB-immunopositive cell bodies and fibres differed between the control and SBA chicks (Fig. 5I, J). In the SBA chicks, the number of CB-immunopositive cells in laminae I-III during the embryonic stage was higher than in normal chicks. However, post-hatching, the number and immunoreactivities of CB-immunopositive neurons were lower in SBA chicks than in normal chicks.

\section{GABAergic transmission in the dorsal horn of the spinal cord}

To determine whether GABAergic neurons were colocalised in spinal cord dorsal horn and the painlike behaviour exhibited by SBA chicks was associated with alterations in GABAergic transmission, double immunofluorescence staining of GABA and $C B$ or $C R$ in dorsal horn laminae I-III of the spinal cord was performed at ED-14 to PD-10. Double staining of GABA and CB (Fig. 6A, B, E, F) or CR (Fig. 6I, J, M, N) increased during the later stages of the embryonic period. This staining was very strong at ED-18 (Fig. 6F, N) but decreased after PD-4 (Fig. 6G, H, O, P), the time point at which the SBA chicks showed severe pain-like behavioural changes (Fig. 1, Suppl. Video 1).

\section{Neurodegeneration in the spinal cord}

To clarify the mechanisms underlying the loss of GABAergic neurons in SBA chicks, the immunoreactivity of caspase-3, a marker of apoptosis, in CBexpressing neurons was evaluated in the dorsal horn laminae I-III of the spinal cord (Fig. 7). In SBA chicks, weak caspase-3 immunoreactivity was evident in CB-expressing neurons at ED-18 (Fig. 7E), and moderate to high caspase- 3 immunoreactivity was found 

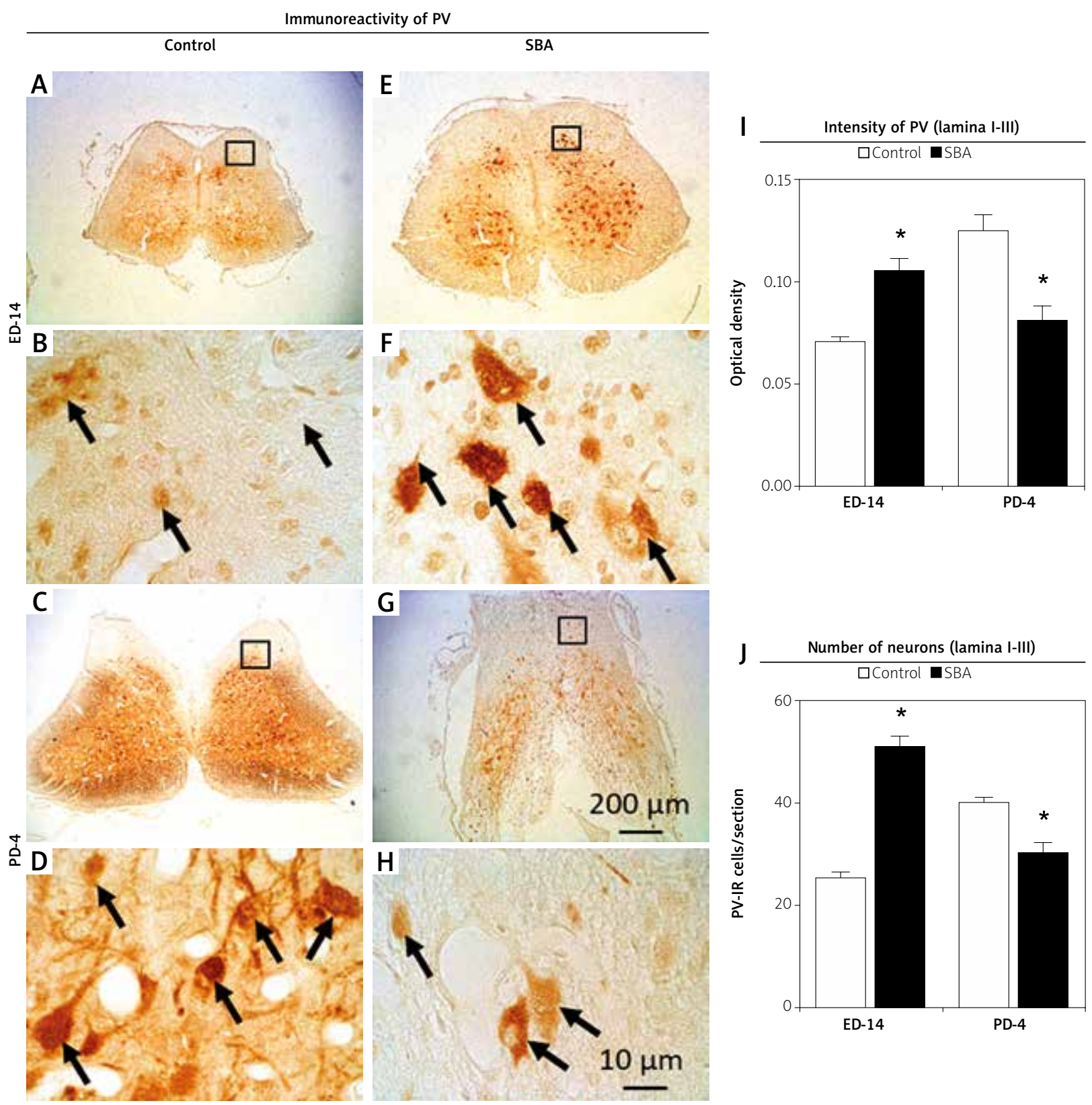

Fig. 3. Postnatal loss of parvalbumin (PV) immunoreactivity in the spinal cord dorsal horns of spina bifida aperta (SBA) chicks. A-H) Immunohistochemical analyses of the expression and localisation of PV in the spinal cord open defect area in SBA chicks, and at a similar location in normal chicks, at ED-14 and PD-4. Representative images of the paraffin-embedded spinal cords of control (A-D) and SBA (E-H) chicks. I) PV immunoreactivity in the dorsal horn laminae I-III of control and SBA chicks measured using ImageJ software. The optical density (OD) was calculated using the following formula: $\mathrm{OD}=$ (log10 [incident light/transmitted light]). Six random sections per chick at each age and in each group were analysed. J) Numbers of PV-immunopositive cells in the spinal cord dorsal horn laminae I-III of control and SBA chicks. The data in Figure $3 \mathrm{I}$ and J are presented as means \pm SEM; $n=6$ per group at each age. ${ }^{*}$ Significantly different from the SBA group at each age $(p<0.01)$, two-way ANOVA and post hoc Tukey's test. Arrows indicate PV-immunopositive cells. 


\begin{tabular}{ll} 
Immunoreactivity of CR & \\
\hline Control & SBA
\end{tabular}
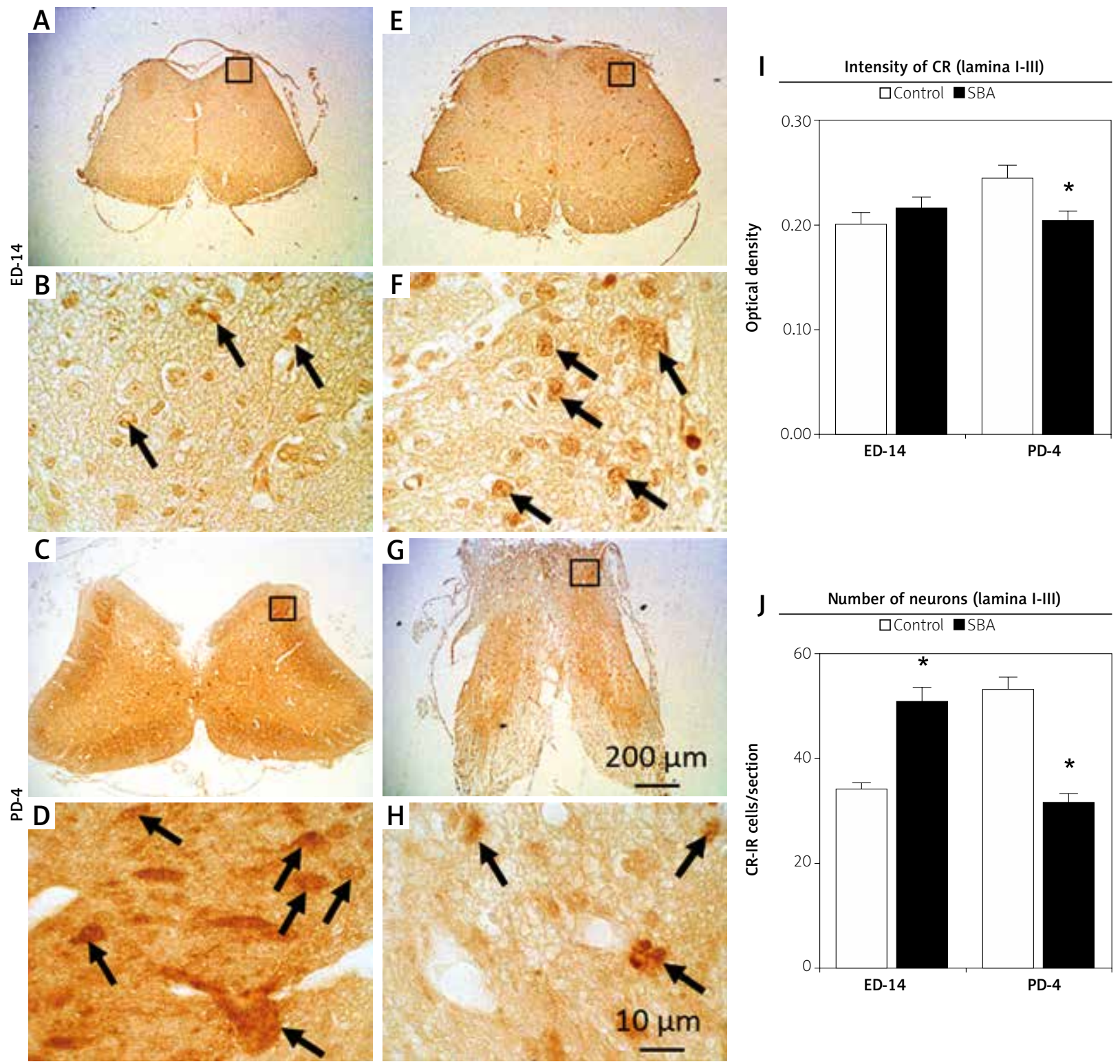

Fig. 4. Postnatal loss of calretinin (CR) immunoreactivity in the spinal cord dorsal horns of spina bifida aperta (SBA) chicks. A-H) Immunohistochemical analyses of the expression and localisation of CR in the spinal cord open defect area in SBA chicks, and at a similar location in normal chicks, at ED-14 and PD-4. Representative images of the paraffin-embedded spinal cords of control (A-D) and SBA (E-H) chicks. I) CR immunoreactivity in the dorsal horn laminae I-III of control and SBA chicks measured using ImageJ software. The optical density (OD) was calculated using the following formula: $O D=(\log 10$ [incident light/transmitted light]). Six random sections per chick at each age and for each group were analysed. J) Numbers of CR-immunopositive cells in the spinal cord dorsal horn laminae I-III of control and SBA chicks. The data in Figure $4 \mathrm{I}$ and $\mathrm{J}$ are means \pm SEM; $n=6$ per group at each age. *Significantly different from the SBA group at each age $(p<0.01)$, twoway ANOVA and post hoc Tukey's test. Arrows indicate CR-immunopositive cells. 


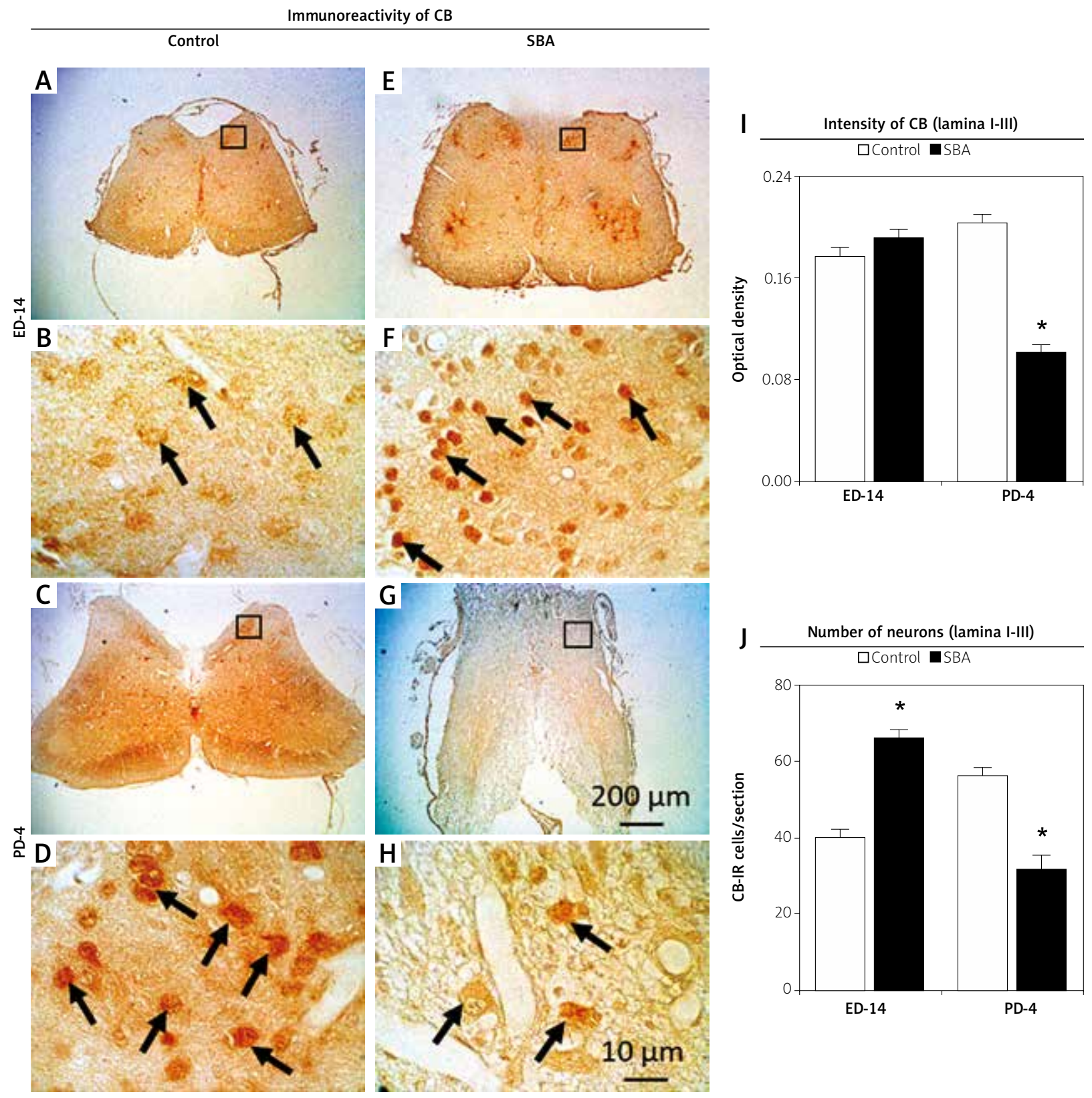

Fig. 5. Postnatal loss of calbindin-D-28K (CB) immunoreactivity in the spinal cord dorsal horns of spina bifida aperta (SBA) chicks. A-H) Immunohistochemical analyses of the expression and localisation of CB in the spinal cord open defect area in SBA chicks, and at a similar location in normal chicks, at ED14 and PD-4. Representative images of the paraffin-embedded spinal cords of control (A-D) and SBA (E-H) chicks. I) $C B$ immunoreactivity in the dorsal horn laminae I-III of control and SBA chicks measured using ImageJ software. The optical density (OD) was calculated using the following formula: OD = (log10 [incident light/ transmitted light]). Six random sections per chick at each age and for each group were analysed. J) Numbers of CB-immunopositive cells in the spinal cord dorsal horn laminae I-III of control and SBA chicks. The data in Figure $5 \mathrm{I}$ and $\mathrm{J}$ are presented as means $\pm \mathrm{SEM} ; n=6$ per group at each age. *Significantly different from the SBA group at each age $(p<0.01)$, two-way ANOVA and post hoc Tukey's test. Arrows indicate CBimmunopositive cells. 


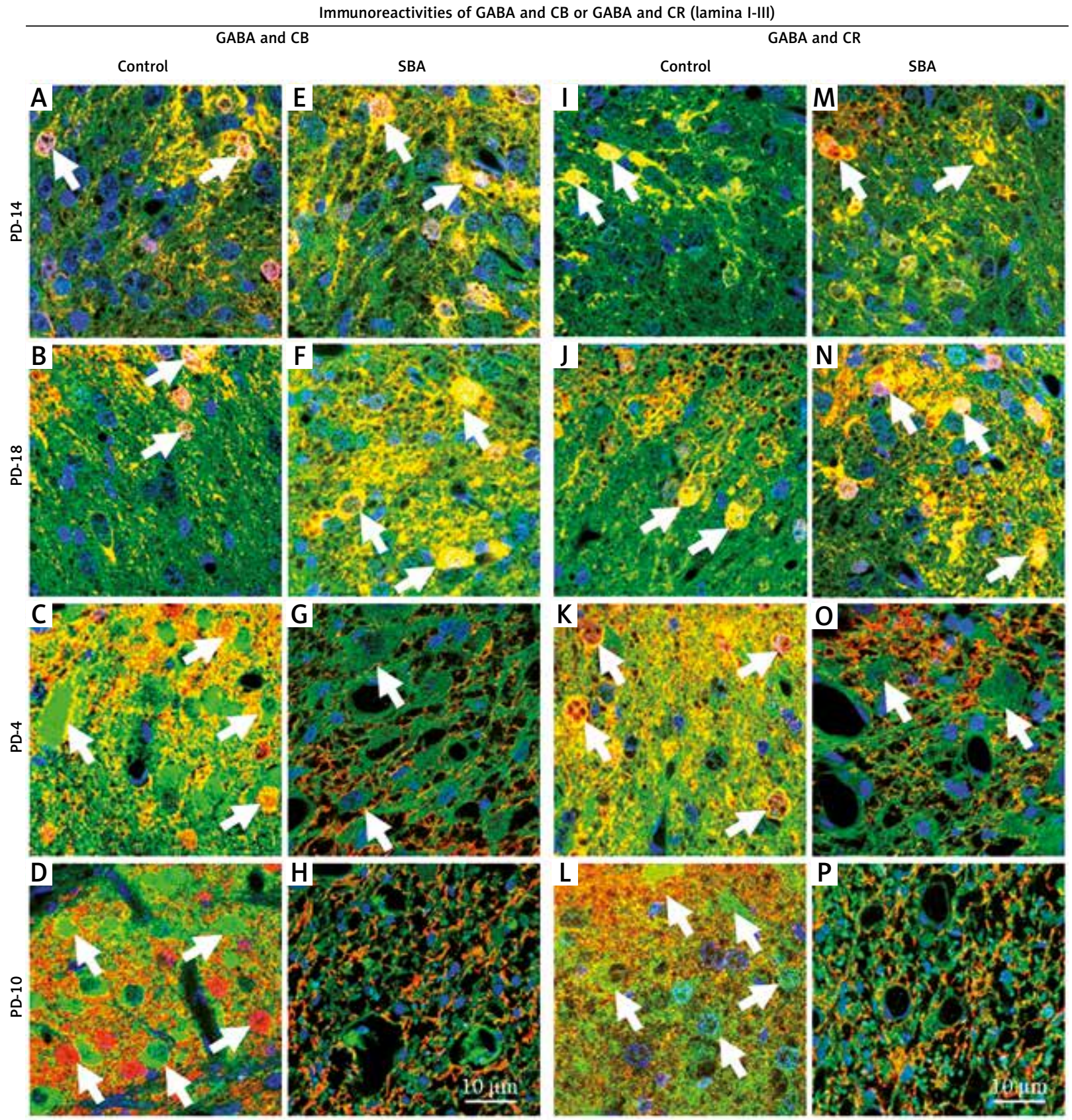

Fig. 6. Postnatal loss of GABAergic transmission in the spinal cord dorsal horns of spina bifida aperta (SBA) chicks. A) Double immunofluorescence staining for GABA and either CB or CR. Representative confocal images of the localisation of GABA and CB (A-H) or GABA and CR (I-P) in normal and SBA chicks on ED-14, ED-18, PD-4, and PD-10. Images of the dorsal horn (laminae I-III) in the open defect of the lumbar cord in SBA chicks and in a similar location in normal chicks. GABA - red; CB or CR - green; co-localisation - yellow; DAPI - blue. Arrows indicate GABAergic-immunopositive cells.

at PD-2 and PD-4 (Fig. 7F, G). However, most of these GABAergic neurons in the dorsal horn had probably degenerated by PD-10, because only a few interneuron-like structures remained at this point (Fig. $7 \mathrm{H}$ ), suggesting that the degeneration of GABAergic neurons in the dorsal horn of the exposed cord of the SBA chicks was due to apoptosis. In addition to the GABAergic neurons, strong caspase-3 immuno- 

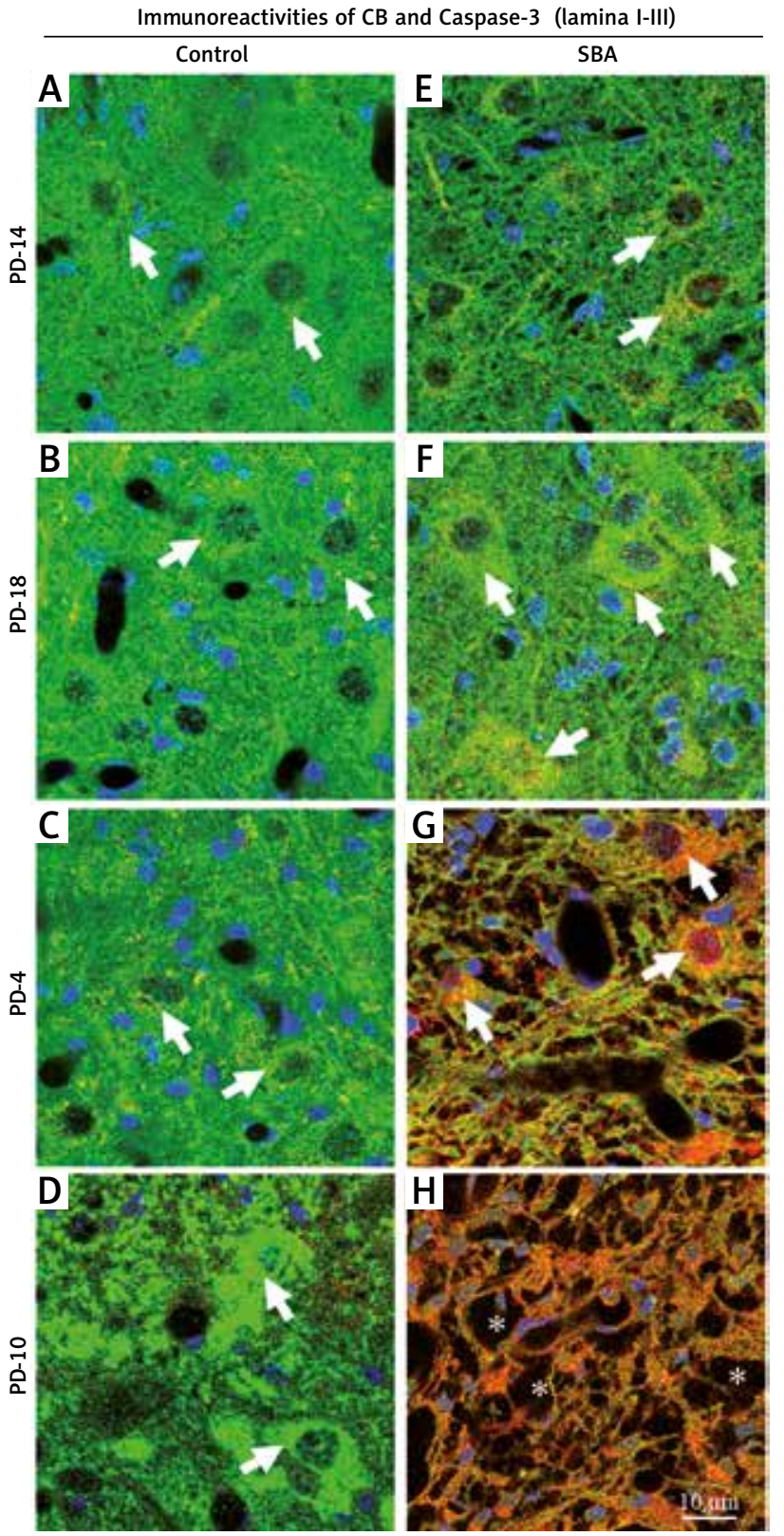

Fig. 7. Caspase 3-mediated apoptosis of GABAergic neurons in the spinal cord dorsal horns of spina bifida aperta (SBA) chicks. A-H) Representative confocal images showing immunofluorescence staining for caspase-3, a marker of apoptosis, and CB-expressing neurons, in the dorsal horn open defect in the lumbar cord of SBA chicks (E-H) and at a similar location in normal chicks (A-D). Caspase-3 - red; CB - green; co-localisation - yellow; DAPI - blue. * Interneuron-like damaged structures in SBA chicks at PD-10. Arrows indicate caspase- 3 immunoreactivity in CB-expressing neurons. reactivity was observed in the tissue of the exposed cord in the SBA chicks at P4 (Fig. 7G), suggesting that the loss of tissue area may also be due to apoptosis.

\section{Discussion}

Chicks with SBA-like features showed various neurological complications, including pain-related behaviour (Fig. 1 and Suppl. Video 1) and motor dysfunction [17], consistent with reports of human neonates with SBA $[35,40,41]$. These behavioural changes in SBA chicks were concomitant with loss of transmission of GABA and its subpopulations PV, $C B$, and $C R$ in the spinal cord dorsal horn. In fact, in contrast to the embryonic period, progressive reduction of GABAergic expression was observed in the spinal dorsal horn of SBA chicks on the neonatal days. Within the first four neonatal days, there was a drastic decrease in GABAergic expression, and this activity was almost absent on P-10 (Figs. 2-6). Furthermore, GABAergic neurons in the spinal cord dorsal horn of SBA chicks underwent apoptosis within the first two weeks after hatching (Fig. 7). Therefore, pathological characterisation of spinal networks of SBA chicks would provide insight into the progression of SBA-like neurological complications.

Spinal cord injury-induced pain complications are due in part to neuronal dysfunction in the spinal cord, characterised by decreasing inhibitory controls $[6,10,22,30]$. In fact, loss of spinal cord dorsal horn inhibitory circuits, many of which involve interneurons that express GABA and its subpopulations, is a major contributor to persistent pain [13-15,23,34,42]. In this study, GABA- and PV-, CB-, and CR-expressing neurons were lost, and the expression levels of these factors were reduced in the exposed spinal cord dorsal horns of SBA chicks at the neonatal stage (Figs. 2-6). Therefore, reduction in GABAergic transmission in the spinal cord dorsal horn may have disrupted the inhibitory networks and contributed to the increased pain exhibited by SBA chicks. This was supported by the abnormal sensory projections in the dorsal funiculi of SBA chicks [38], which is the main route of inhibitory pain pathways in the spinal cord [27]. Although it is not possible to quantify pain in chicks, our behavioural observations suggested that the SBA chicks experienced persistent pain. The SBA chicks produced an increased number of loud vocalisations (Fig. 2B, C) and exhibited reduced confidence in mobility, escape 
reactions, anxiety, fear or restlessness, and struggling to change position (Suppl. Video 1), major indicators of pain in birds [25,33]. Thus, suppression of GABAergic transmission in the superficial dorsal horn may cause SBA-related pain.

Our findings also support a possible correlation between the loss of GABAergic neurons and neurological disorders in SBA patients, because intrathecal treatment with a GABA agonist relieves SBA-induced pain and spasticity in humans [5]. This also indicates that the reduction in spinal GABAergic transmission in the motor neuron area observed in our previous study contributed to leg movement dysfunction in the SBA chicks [17]. The loss of GABAergic transmission from the dorsal and ventral horn is also supported by the decreased or absent sensorimotor function in neonatal SBA chicks (Table I). Taken together, these findings provide strong pathophysiological evidence that the loss of GABAergic transmission leads to varying degrees of motor and/or sensory deficits in the spinal cord, and that this contributed to various neurological complications, particularly leg movement dysfunction and pain, in SBA chicks.

In this study, increases in GABAergic immunoreactivities were found in spinal dorsal horn of SBA chicks at midgestation, and these activities were very strong at advanced stages (Figs. 2-6). The widespread GABAergic immunoreactivity in the embryonic period indicates that this neurotransmitter system may play a neuroprotective role against amniotic fluid-induced neurotoxicity and/or other neurotoxic insults, as has been observed in the spinal tissues [17,23]. Also, we cannot exclude the possibility that excess GABA, via the GABA shunt, may be a source of energy for abnormal neurons in the exposed cord area to promote their proliferation and migration, as has been observed in various neoplastic cells $[31,39]$. These possibilities are supported by our results because SBA chicks had increased numbers of GABAergic neurons in advanced gestational stages (Figs. 2-5). Although further studies are needed to clarify the actual roles that increased GABAergic activities play, the present results indicate that the functionality of GABAergic neurons in spinal dorsal horn of SBA chicks is compromised during the embryonic period starting from at least midgestation.

Neuronal loss due to apoptosis is an important pathophysiological component of many neurological diseases $[16,21,37]$. The loss of spinal neurons at lesion sites may be associated with SBA-related neurological dysfunctions [1]. In fact, Kowitzk et al. suggested that neurons in neonatal myelomeningoceles undergo degeneration by apoptosis [20]. Apoptosis-induced degeneration of GABAergic interneurons and motor neurons in the lumbar cord ventral horn area is reportedly associated with SBA-like motor dysfunctions [17]. In this study, caspase-3 immunoreactivity - a marker of apoptosis - in GABAergic neurons in the dorsal horns of SBA chicks was evident at an advanced gestational stage (ED-18). Caspase-3 immunoreactivity gradually increased at the early neonatal stage and was strong at PD-10, when SBA chicks exhibited only a few damaged interneuron-like structures in the dorsal horn area (Fig. 7). Collectively, these findings demonstrate that reduced inhibitory transmission and increased apoptotic activity in the exposed spinal cord are involved in the pathogenesis of neurological complications, such as motor deficits and pain, in SBA chicks.

In conclusion, our findings provide evidence that early neonatal loss of inhibitory transmission disrupts the neuronal networks in the spinal cord dorsal horn, and that this contributes to SBA-related pain-like complications. Moreover, our results shed light on the mechanisms underlying cellular abnormalisation and degeneration. They also suggest that the effects of neurological disorders in SBA patients can be modulated by manipulating the GABAergic system, which may be a promising avenue for novel therapeutic interventions.

\section{Acknowledgements}

We would like to thank D. Shimizu for his technical support with confocal imaging.

This work was supported in part by grants to M.S.I.K. from the Japan Society for the Promotion of Science (No. 15K20005 and 18K07500) and to S.M (No. 18K08945).

\section{Disclosure}

The authors report no conflict of interest.

\section{References}

1. Adzick NS. Fetal surgery for myelomeningocele: trials and tribulations. Isabella Forshall Lecture. J Pediatr Surg 2012; 47: 273-281.

2. Alriksson-Schmidt AI, Thibadeau JK, Swanson ME, Marcus D, Carris KL, Siffel C, Ward E. The natural history of spina bifida in children pilot project: research protocol. JMIR Res Protoc 2013; 2: e2. 
3. Antal M, Freund TF, Polgar E. Calcium-binding proteins, parvalbumin- and calbindin-D 28k-immunoreactive neurons in the rat spinal cord and dorsal root ganglia: a light and electron microscopic study. J Comp Neurol 1990; 295: 467-484.

4. Arvidsson U, Ulfhake B, Cullheim S, Ramírez V, Shupliakov O, Hökfelt T. Distribution of calbindin D28k-like immunoreactivity (LI) in the monkey ventral horn: do Renshaw cells contain calbindin D28k-LI? J Neurosci 1992; 12: 718-728.

5. Bergenheim AT, Wendelius M, Shahidi S, Larsson E. Spasticity in a child with myelomeningocele treated with continuous intrathecal baclofen. Pediatr Neurosurg 2003; 39: 218-221.

6. Castro-Lopes JM, Tavares I, Coimbra A. GABA decreases in the spinal cord dorsal horn after peripheral neurectomy. Brain Res 1993; 620: 287-291.

7. Clancy CA, McGrath PJ, Oddson BE. Pain in children and adolescents with spina bifida. Dev Med Child Neurol 2005; 47: 27-34.

8. Copp AJ, Adzick NS, Chitty LS, Fletcher JM, Holmbeck GN, Shaw GM. Spina bifida. Nat Rev Dis Primers 2015; 1: 15007.

9. Copp AJ, Brook FA, Estibeiro JP, Shum AS, Cockroft DL. The embryonic development of mammalian neural tube defects. Prog Neurobiol 1990; 35: 363-403.

10. Coull JA, Boudreau D, Bachand K, Prescott SA, Nault F, Sík A, De Koninck P, De Koninck Y. Trans-synaptic shift in anion gradient in spinal lamina I neurons as a mechanism of neuropathic pain. Nature 2003; 424: 938-942.

11. Hamburger V, Hamilton HL. A series of normal stages in the development of the chick embryo. J Morphol 1951; 88: 49-92.

12. Heffez DS, Aryanpur J, Hutchins GM, Freeman JM. The paralysis associated with myelomeningocele: clinical and experimental data implicating a preventable spinal cord injury. Neurosurgery 1990; 26: 987-992.

13. Hiura A, Nasu F, Ishizuka H, Kuwahara M, Ohta M. Evidence of synaptic contacts of nociceptive primary afferent central terminals on GABAergic interneurons in the substantia gelatinosa. Arch Histol Cytol 1996; 59: 55-60.

14. Ibuki T, Hama AT, Wang XT, Pappas GD, Sagen J. Loss of GABAimmunoreactivity in the spinal dorsal horn of rats with peripheral nerve injury and promotion of recovery by adrenal medullary grafts. Neuroscience 1997; 76: 845-858.

15. Jelsma F, Ploetner EJ. Painful spina bifida occulta; with review of the literature. J Neurosurg 1953; 10: 19-27.

16. Keane RW, Kraydieh S, Lotocki G, Alonso OF, Aldana P, Dietrich WD. Apoptotic and antiapoptotic mechanisms after traumatic brain injury. J Cereb Blood Flow Metab 2001; 21: 11891198.

17. Khan MSI, Nabeka H, Islam F, Shimokawa T, Saito S, LiX, Kawabe S, Hamada F, Tachibana T, Matsuda S. Early neonatal loss of inhibitory synaptic input to the spinal motor neurons confers spina bifida-like leg dysfunction in a chicken model. Dis Model Mech 2017; 10: 1421-1432.

18. Kinutani M, Coltey M, Le Douarin NM. Postnatal development of a demyelinating disease in avian spinal cord chimeras. Cell 1986; 45: 307-314.

19. Kinutani M, Le Douarin NM. Avian spinal cord chimeras: I. Hatching ability and posthatching survival in homo- and heterospecific chimeras. Dev Biol 1985; 111: 243-255.
20. Kowitzke B, Cohrs G, Leuschner I, Koch A, Synowitz M, Mehdorn HM, Held-Feindt J, Knerlich-Lukoschus F. Cellular profiles and molecular mediators of lesion cascades in the placode in human open spinal neural tube defects. J Neuropathol Exp Neurol 2016; 75: 827-842.

21. Lawson SJ, Lowrie MB. The role of apoptosis and excitotoxicity in the death of spinal motoneurons and interneurons after neonatal nerve injury. Neuroscience 1998; 87: 337-348.

22. Lever IJ, Pezet S, McMahon SB, Malcangio M. The signaling components of sensory fiber transmission involved in the activation of ERK MAP kinase in the mouse dorsal horn. Mol Cell Neurosci 2003; 24: 259-270.

23. Liu J, Wolfe D, Hao S, Huang S, Glorioso JC, Mata M, Fink DJ. Peripherally delivered glutamic acid decarboxylase gene therapy for spinal cord injury pain. Mol Ther 2004; 10: 57-66.

24. Malcangio M, Bowery NG. GABA and its receptors in the spinal cord. Trends Pharmacol Sci 1996; 17: 457-462.

25. Malik A, Valentine A. Pain in birds: a review for veterinary nurses. Vet Nurs J 2018; 33: 11-24.

26. Meuli M, Meuli-Simmen C, Hutchins GM, Yingling CD, Hoffman KM, Harrison MR, Adzick NS. In utero surgery rescues neurological function at birth in sheep with spina bifida. Nat Med 1995; 1: 342-347.

27. Millan MJ. Descending control of pain. Prog Neurobiol 2002; 66: 355-474.

28. Millicovsky G, Lazar ML. Spina bifida: role of neural tissue damage during pregnancy in producing spinal paralysis. Obstet Gynecol 1995; 86: 300-301.

29. Mominoki K, Kinutani M, Wakisaka H, Saito S, Kobayashi N, Fujiwara T, Matsuda S. Leg dysfunctions in a hatched chick model of spina bifida aperta. Exp Neurol 2006; 197: 133-142.

30. Moore KA, Kohno T, Karchewski LA, Scholz J, Baba H, Woolf CJ. Partial peripheral nerve injury promotes a selective loss of GABAergic inhibition in the superficial dorsal horn of the spinal cord. J Neurosci 2002; 22: 6724-6731.

31. Neman J, Termini J, Wilczynski S, Vaidehi N, Choy C, Kowolik CM, Li H, Hambrecht AC, Roberts E, Jandial R. Human breast cancer metastases to the brain display GABAergic properties in the neural niche. Proc Natl Acad Sci U S A 2014; 111: 984-989.

32. Osaka K, Tanimura T, Hirayama A, Matsumoto S. Myelomeningocele before birth. J Neurosurg 1978; 49: 711-724.

33. Prunier A, Mounier L, Le Neindre P, Leterrier C, Mormède P, Paulmier V, Prunet P, Terlouw C, Guatteo R. Identifying and monitoring pain in farm animals: a review. Animal 2013; 7: 998-1010.

34. Rausell E, Cusick CG, Taub E, Jones EG. Chronic deafferentation in monkeys differentially affects nociceptive and nonnociceptive pathways distinguished by specific calcium-binding proteins and down-regulates gamma-aminobutyric acid type A receptors at thalamic levels. Proc Natl Acad Sci U S A 1992; 89: 2571-2575.

35. Sival DA, van Weerden TW, Vles JS, Timmer A, den Dunnen WF, Staal-Schreinemachers AL, Hoving EW, Sollie KM, KranenMastenbroek VJM, Sauer PJJ, Brouwer OF. Neonatal loss of motor function in human spina bifida aperta. Pediatrics 2004; 114: 427-434.

36. Szymanski KM, Cain MP, Whittam B, Kaefer M, Rink RC, Misseri R. Incontinence affects health-related quality of life in children 
and adolescents with spina bifida. J Pediatr Urol 2018; 14: 279. e1-279.e8.

37. Tatton WG, Chalmers-Redman RM, Ju WY, Wadia J, Tatton NA. Apoptosis in neurodegenerative disorders: potential for therapy by modifying gene transcription. J Neural Transm Suppl 1997; 49: 245-268.

38. Tsujimura R, Mominoki K, Kinutani M, Shimokawa T, Doihara T, Nabeka H, Wakisaka H, Kobayashi N, Matsuda S. Sensory tract abnormality in the chick model of spina bifida. Neurosci Res 2011; 71: 85-91.

39. Van Swearingen AE, Siegel MB, Anders CK. Breast cancer brain metastases: evidence for neuronal-like adaptation in a 'breastto-brain' transition? Breast Cancer Res 2014; 16: 304.

40. Werhagen L, Gabrielsson H, Westgren N, Borg K. Medical complication in adults with spina bifida. Clin Neurol Neurosurg 2013; 115: 1226-1229.

41. Werhagen L, Hultling C, Borg K. Pain, especially neuropathic pain, in adults with spina bifida, and its relation to age, neurological level, completeness, gender and hydrocephalus. Rehabil Med 2010; 42: 374-376.

42. Zhang AL, Hao JX, Seiger A, Xu XJ, Wiesenfeld-Hallin Z, Grant G, Aldskogius $H$. Decreased GABA immunoreactivity in spinal cord dorsal horn neurons after transient spinal cord ischemia in the rat. Brain Res 1994; 656: 187-190. 